\title{
息 Jefferson.
}

\section{The Medicine Forum}

Volume 13

Article 6

2012

\section{Non-ST Elevation MI as a Unique Presentation of Angioimmunoblastic T-cell Lymphoma}

Paurush Shah, MD

Thomas Jefferson University

Gunjan Shah, MD

Thomas Jefferson University

Avinash Chandra, MD

Thomas Jefferson University

Lawrence Lee, MSIV

Thomas Jefferson University

Gregary Marhefka, MD

Thomas Jefferson University

Follow this and additional works at: https://jdc.jefferson.edu/tmf

Part of the Medicine and Health Sciences Commons

Let us know how access to this document benefits you

\section{Recommended Citation}

Shah, MD, Paurush; Shah, MD, Gunjan; Chandra, MD, Avinash; Lee, MSIV, Lawrence; and Marhefka, MD, Gregary (2012) "Non-ST Elevation MI as a Unique Presentation of Angioimmunoblastic T-cell Lymphoma," The Medicine Forum: Vol. 13 , Article 6. DOI: https://doi.org/10.29046/TMF.013.1.007

Available at: https://jdc.jefferson.edu/tmf/vol13/iss1/6

This Article is brought to you for free and open access by the Jefferson Digital Commons. The Jefferson Digital Commons is a service of Thomas Jefferson University's Center for Teaching and Learning (CTL). The Commons is a showcase for Jefferson books and journals, peer-reviewed scholarly publications, unique historical collections from the University archives, and teaching tools. The Jefferson Digital Commons allows researchers and interested readers anywhere in the world to learn about and keep up to date with Jefferson scholarship. This article has been accepted for inclusion in The Medicine Forum by an authorized administrator of the Jefferson Digital Commons. For more information, please contact: JeffersonDigitalCommons@jefferson.edu. 


\title{
Non-ST Elevation Mi as a Unique Presentation of ANGIOIMMUNOBLASTIC T-CELL LYMPHOMA
}

\author{
Paurush Shah, MD, Gunjan Shah, MD, Avinash Chandra, MD, Lawrence Lee, MSIV, and Gregary Marhefka, MD
}

\section{Case}

A 61-year-old Chinese female with a history of hypertension, hyperlipidemia, asthma, and gastroesophageal reflux disease presented with four days of chest pressure that radiated to her left arm and jaw. On exam, her vital signs were within normal limits and cardiac and pulmonary exams were unremarkable. Her initial electrocardiogram (ECG) demonstrated ischemic ST segment depressions in leads II, III, and aVF; her first troponin I was elevated at $2.3 \mathrm{ng} / \mathrm{mL}$ (normal $<0.05 \mathrm{ng} / \mathrm{mL}$ ) and peaked at $6.8 \mathrm{ng} / \mathrm{mL}$. She was given sublingual nitroglycerin and metoprolol, which controlled her symptoms, and she was started on a heparin infusion to prevent further ischemia. The patient's medications included: amlodipine was notable for the following daily medications: amlodipine $5 \mathrm{mg}$, olmesartan $20 \mathrm{mg}$, atorvastatin $20 \mathrm{mg}$, esomeprazole $20 \mathrm{mg}$, montelukast $10 \mathrm{mg}$, and mometasone $110 \mathrm{mcg}$ twice daily. Her family history was significant for a brother who had a coronary stent placed at age 57. She denied drug, tobacco, or alcohol use. She was non-English speaking, and immigrated to the United States from China five years ago.

The day after she presented to the hospital, she underwent cardiac catheterization, which revealed diffuse coronary artery disease with the following arterial stenoses: $50 \%$ distal left anterior descending (LAD) and 50\% mid right coronary artery (RCA) with a $100 \%$ distal vessel supplied by extensive left to right collaterals. No interventions were performed. An echocardiogram (echo) was performed which noted equivocal apical septal hypokinesis. Of note, there were circular echodensities with a central echo-free space in the left and right atrioventricular grooves of unclear significance. Cardiac gated computed tomography angiography (CTA) was recommended as an outpatient. The patient was medically managed for a non-ST elevation MI (NSTEMI) and discharged home. Further evaluation of the atrioventricular densities seen on echocardiogram was to be done as an outpatient.

Approximately three weeks later, the patient presented again to the emergency department with recurrent angina, which awakened her from sleep. The ambulance ECG was notable for dynamic ST segment depression, though her troponin was initially within normal limits. The patient was medically stabilized and cardiac catheterization was urgently performed, which again revealed marked progression of coronary stenoses: $90 \%$ mid and $50 \%$ distal LAD, 90\% first diagonal, 90\% distal left circumflex, $90 \%$ first obtuse marginal, $90 \%$ third obtuse marginal, $70 \%$ mid RCA, and subtotaled distal right coronary now with right to left collaterals. A total of $300 \mathrm{mcg}$ of intracoronary nitroglycerin was infused with no change in the stenoses. No other interventions were performed and the patient was aggressively treated for coronary vasospasm with diltiazem and isosorbide mononitrate. Her metoprolol was discontinued. Two days later, while medically stable, she underwent a repeat cardiac catheterization, which now revealed resolution of the coronary vasospasm with the same stenoses as her original cardiac catheterization three weeks prior. A rheumatologic work-up was initiated given the unusual atrioventricular densities seen on echocardiogram with vasospasm, as this may have suggested a possible vasculitis. The patient was discharged home again with close follow-up while the rheumatology labs were pending.

Three weeks later, the patient presented again with early morning angina and was treated medically for coronary vasospasm, as well as a recurrent NSTEMI. The patient was stabilized and a cardiac gated CTA was performed, which revealed sleeve-like soft tissue densities encasing the right and left circumflex coronary arteries as well as the proximal portion of the LAD (Figure 1). The prior rheumatologic work-up revealed only a modestly elevated erythrocyte sedimentation rate (ESR). Rheumatology was consulted and they suggested biopsy for tissue diagnosis though one was not performed at that time. A computed tomography (CT) of the chest, abdomen, and pelvis was done revealing mediastinal and para-aortic lymph nodes. A cardiac gated magnetic resonance imaging (MRI) was performed which further characterized the known atrioventricular soft tissue densities (Figure 2). On hospital day 9, the patient developed late evening angina with recurrent ECG ST depressions, and despite sublingual nitroglycerin and eventually an intravenous infusion, the patient became rapidly hypotensive, lost her pulse and suffered a pulseless electrical activity cardiac arrest with failure to respond to resuscitation.

The family agreed to an autopsy which revealed angioimmunoblastic T-cell lymphoma (AITL) with involvement of the coronary epicardium and nodular perivascular infiltration of the right mainstem, left circumflex, and left anterior descending coronary arteries, kidneys, and spleen, as well as mediastinal, omental, mesenteric and para-aortic lymph nodes. (Figure 3 A-D). Pathology revealed the classic picture for AITL, polymorphic infiltrate of lymphocytes, plasma cells, and eosinophils (Figure 4B). Other findings included proliferation of venules and follicular dendritic cells, monoclonal/oligoclonal T-cells present (CD3+/ CD4+), and sometimes oligoclonal or monoclonal B-cell populations attributed to B-cells infected with Epstein-Barr virus (EBV) (Figure $4 \mathrm{~A}-\mathrm{C}$ ).

\section{Discussion}

Recurrent NSTEMIs secondary to coronary vasospasm with echodense masses seen in the atrioventricular grooves is a unique presentation with a varied differential including malignancies and rheumatic diseases. More frequent cardiac imaging and biochemical laboratory testing have increased the incidence and prevalence of cardiovascular diseases found in patients with rheumatic disease. ${ }^{1}$ Systemic lupus erythematosus, 


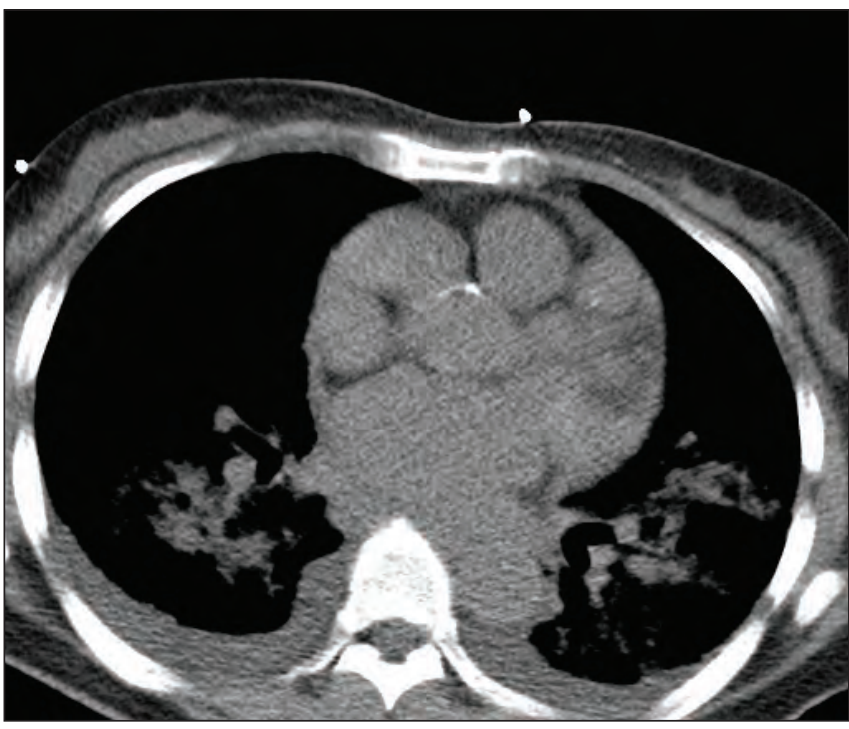

Figure 1. CT showing sleeve-like densities surrounding coronaries

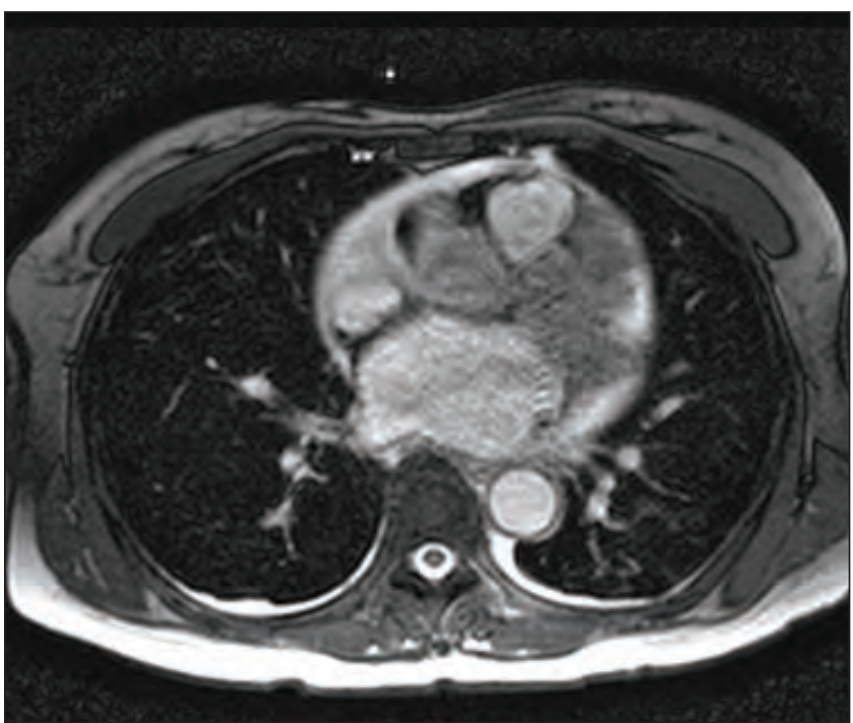

Figure 2. MRI show sleeve-like densities surrounding coronaries

Takayasu disease, rheumatoid arthritis, polyarteritis nodosa, Churg-Strauss syndrome, Behçet's disease, sarcoidosis, and giant cell arteritis are some of the many reported causes of coronary artery vasculitis. ${ }^{2,3}$ Additionally, a case of lymphoplasmacytic arteritis of the coronary and internal mammary arteries with no systemic disease has also been documented. ${ }^{3}$ Myocardial ischemia and infarction without serological evidence of inflammatory disease, as in this patient, unfortunately do not have established management strategies since revascularization in such cases is often unsuccessful. ${ }^{3}$

Tumors with cardiac involvement may affect all three layers of the heart. Metastatic neoplasms commonly involve the epicardium or pericardium, while the less common primary cardiac malignancies often involve the myocardium or cardiac chambers. ${ }^{4}$
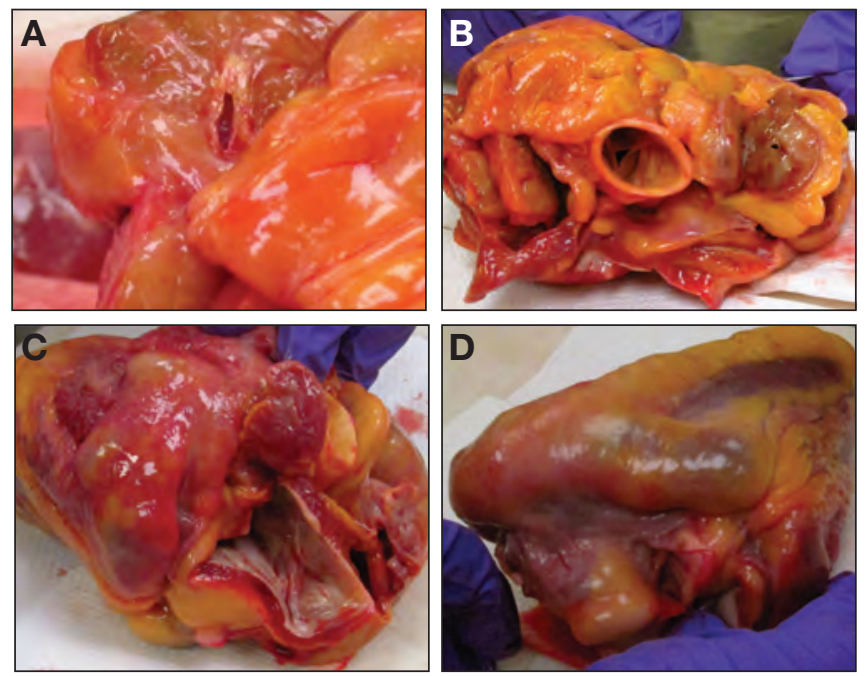

Figure 3. (A) Oblique section through the left circumflex coronary artery; (B) Cross section through the right coronary artery; (C) Severely enlarged left circumflex coronary artery; (D) Severely enlarged right coronary artery

The most common primary benign masses of the heart include myxoma, rhabdomyoma, fibroma, hemangioma, and atrioventricular nodal, while the most common malignant neoplasms include sarcomas (angio, unclassified, fibrous histocytoma, and osteo) and lymphomas. Cardiac involvement from melanoma, germ cell, leukemia, lymphoma, and lung cancers occur in up to $45 \%$ of patients with cardiac metastasis, though these may go unrecognized until autopsy. ${ }^{4,5}$ Direct extension, hematogenous spread, retrograde lymphatic extension, or transvenous extension are the accepted mechanisms of metastasis. Symptoms are based on the tumor's location and often include shortness of breath or chest pain, but most commonly patients present with a pericardial effusion with tamponade. ${ }^{5}$ A preliminary diagnosis can be made by echocardiography; CT and MRI are used for further classification, and biopsy is required for confirmation. Treatment of the primary malignancy, whether surgical excision or medical management, depends upon the tumor classification.

AITL, though rare, is one of the more commonly occurring peripheral T-cell lymphomas and accounts for 2-5\% of all non-Hodgkin's lymphomas. ${ }^{6}$ It arises from peripheral CD4 positive T-cells thought to be a subset of follicular helper T-cells. Patients tend to be diagnosed after a subacute illness in the sixth or seventh decade with a slight male predominance. ${ }^{7}$ Presenting signs and symptoms include asymptomatic generalized lymphadenopathy, fevers, night sweats, arthralgias, and weight loss. Extra-nodal involvement is common and usually includes hepatomegaly, splenomegaly, pleural and pericardial effusions, and various skin rashes. Laboratory abnormalities include an elevated serum lactate dehydrogenase level, a polyclonal hypergammaglobulinemia, and Coombs positive autoimmune hemolytic anemia. ${ }^{6}$ Diagnosis is made by excisional tissue biopsy. Lymph node histology will show an effaced nodal architecture, prominent arborizing high endothelial venules, and 

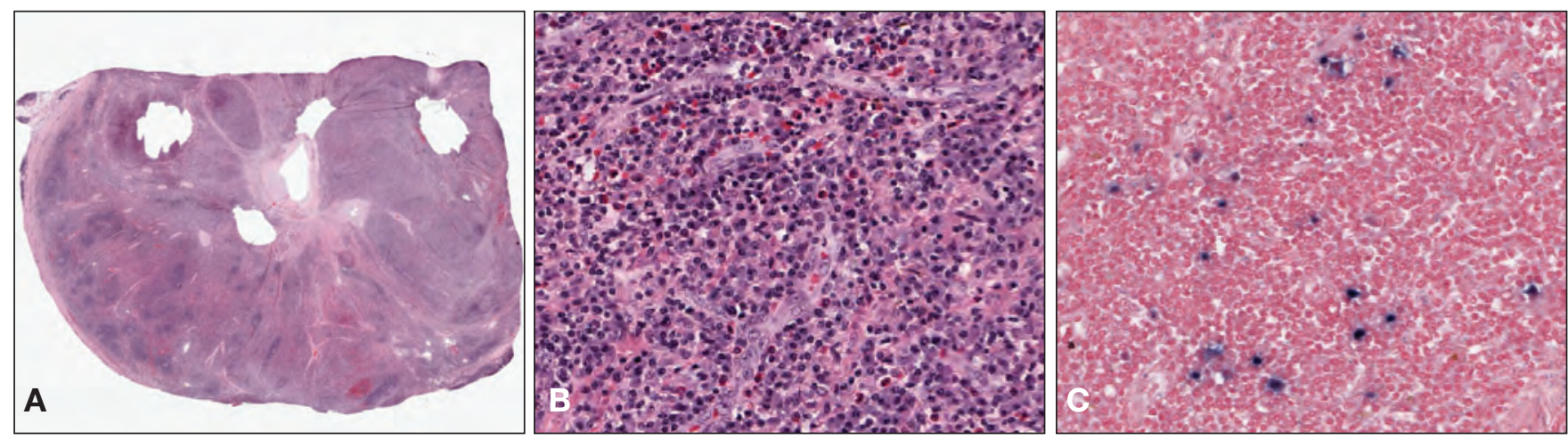

Figure 4. (A) Low power photomicrograph cross section of the RCA. Tumor cells encase the artery as seen on CT and MRI; (B) High power photomicrograph of the RCA showing pleomorphic infiltrate of plasma cells, eosinophils, lymphocytes, and prominence of venules; (C) Cells staining dark blue (positive for EBER, a test for EBV)

a polymorphous infiltrate, which includes reactive lymphocytes, immunoblasts, plasma cells, and atypical lymphocytes positive for CD3 and CD4. ${ }^{7}$ Therapeutic options have not been standardized, but responses have been reported in those receiving single agent corticosteroids, low-dose methotrexate, fludarabine, lenalidomide, and others. ${ }^{6}$ Combination chemotherapy, including regimens such as vincristine, doxorubicin, and prednisone with or without cyclophosphamide, has failed to increase survival rates to greater than $30 \%{ }^{6}$

Upon performing a literature review for AITL, we found no other cases in which the presenting symptom was a myocardial infarction. For extranodal manifestations of AITL, there is a case of syncope due to sinoatrial node disease. ${ }^{5}$ The authors concluded that the syncope was associated with sinus arrest due to ischemic damage and fibrosis of the sinoatrial node secondary to AITL. Our patient's diagnosis was especially challenging given that her workup only demonstrated non-palpable lymphadenopathy and echodense masses in the atrioventricular grooves. These masses caused extrinsic compression of the coronary vasculature resulting in myocardial ischemia, repeated infarction, and ultimately to her death.

\section{References}

1. Maksimovic R, Seferovic PM, Ristic AD, et al. Cardiac imaging in rheumatic diseases. Rheumatology (Oxford) 2006;45 Suppl 4:iv26-31.

2. Kojima M, Nakamura N, Tsukamoto N, et al. Atypical lymphoplasmacytic and immunoblastic proliferation of autoimmune disease : clinicopathologic and immunohistochemical study of 9 cases. J Clin Exp Hematop;50:113-9.

3. Manek NJ, Edwards WD. At the heart of the matter: the exceptional case of coronary artery disease in a young man. Arthritis Rheum 2008;59:1676-81.

4. Roberts WC. Neoplasms involving the heart, their simulators, and adverse consequences of their therapy. Proc (Bayl Univ Med Cent) 2001;14:358-76.

5. Dolan G, Jones AP, Reilly JT. Immunoblastic lymphoma presenting with syncope due to sinoatrial node disease. Postgrad Med J 1990;66:395-7.

6. Alizadeh AA, Advani RH. Evaluation and management of angioimmunoblastic T-cell lymphoma: a review of current approaches and future strategies. Clin Adv Hematol Oncol 2008;6:899-909.

7. de Leval L, Gisselbrecht C, Gaulard P. Advances in the understanding and management of angioimmunoblastic T-cell lymphoma. Br J Haematol 2010;148:673-89. 